\title{
Short-Term Reduction in Energy Availability Does Not Impair Exercise- Induced Gains in Bone Formation Rate
}

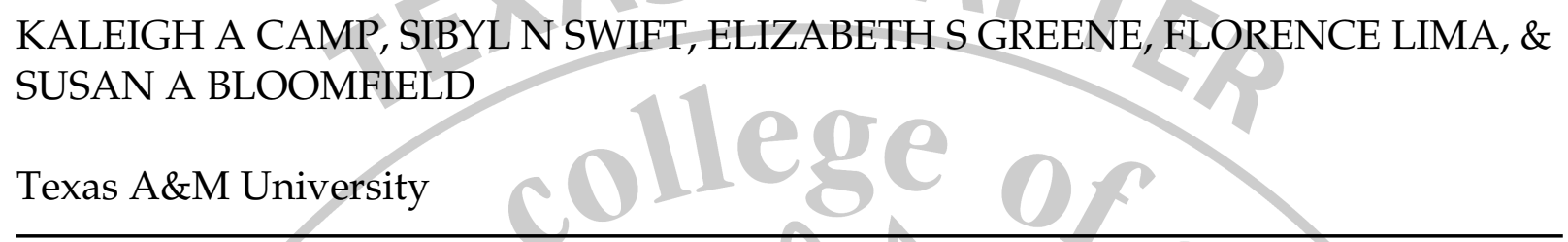

ABSTRACT

Reduced energy availability (EA, defined as total energy intake minus exercise energy expenditure) can induce significant bone loss in humans and in rodents, but this effect may vary with exercise status. Purpose: This study focused on the effects of graded reductions in EA achieved with and without exercise on mid-shaft tibia cortical bone. We hypothesized that markers of bone formation would be reduced with 4 weeks of decreased EA, but these reductions would be mitigated in exercising animals. Methods: After 8 weeks of acclimation to AIN-93M purified diet, 5 mo-old virgin female SpragueDawley rats $(n=72)$ were randomized in to sedentary (SED) and exercise (EX) groups, each of which was divided into 3 energy status groups: $-12 \%$ EA/g body mass (-12EA) and -25\% EA/g body mass (-25EA) vs. ad lib-fed controls. EX rats were restricted on diet intake slightly less than SED to account for the energy cost ( $+10 \% /$ day) of treadmill running $(80-90 \mathrm{~min} / \mathrm{d}, 4 \mathrm{~d} / \mathrm{wk}, \sim 60 \% \mathrm{VO} 2 \mathrm{max})$; custom versions of AIN-93M were used to assure restriction of only kcal (other nutrients at $100 \%$ ). Calcein injections 9 and 2 days prior to euthanasia labeled mineralizing surfaces for histomorphometric analyses $2 \mathrm{~mm}$ proximal to the tibio-fibular junction for mineralizing surface (\%MS/BS), mineral apposition rate (MAR), and bone formation rate (BFR) on periosteal and endocortical surfaces. Results: After 4 weeks, only -25EA SED lost body mass (-11\%); virtually all of this was fat mass. Periosteal BFR was 2 to 4 fold higher in EX rats vs. SED animals within each energy status group. The impact of EX on endocortical BFR was even greater ( $\sim 5$-fold increase) for all groups except in the -25 EA cohorts (-25 EA EX BFR equal to -25EA SED BFR). Increases in BFR with EX were achieved by increases in both \%MS/BS and MAR on both cortical surfaces. Conclusions: These data suggest that short-term graded reductions in EA do not inhibit BFR in SED animals nor the robust stimulation of BFR by moderately vigorous exercise training. The one exception observed was a suppression of the EX induced gain in endocortical BFR with the more stringent level of reduced EA. Whether this lack of effect of EA on BFR gains with EX persists with more chronic reductions in EA has yet to be determined.

Funded by the Department of Defense \#WSIXWH-06-1-0479

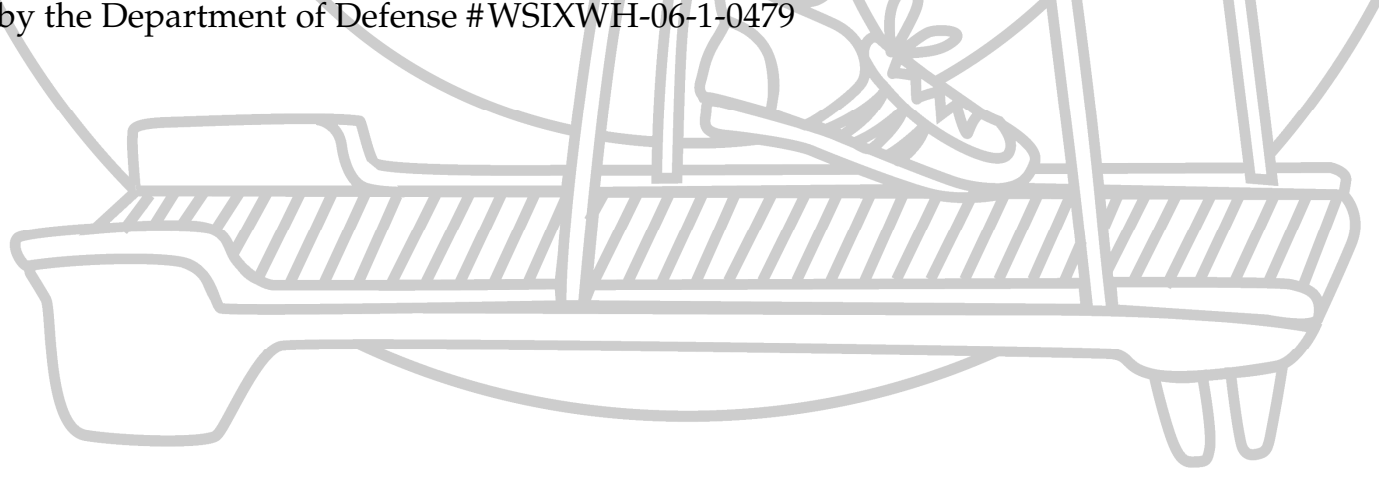

